\title{
South African National Survey of Arachnida (SANSA): Solifugae (sun-spiders) of the national parks and reserves of South Africa (Arachnida, Solifugae)
}

\author{
A. S. DippenaAR-Schoeman and A. X. González Reyes
}

\begin{abstract}
Dippenaar-Schoeman, A.S. and A.X. González Reyes. 2006 South African National Survey of Arachnida (SANSA): Solifugae (sun-spiders) of the national parks and reserves of South Africa (Arachnida, Solifugae). Koedoe 49(2): 29-38. Pretoria. ISSN 0075-6458.
\end{abstract}

\begin{abstract}
As part of the South African National Survey of Arachnida (SANSA) surveys are underway to determine the diversity of the Arachnida fauna of South Africa. One such a project is to make an inventory of the arachnids in protected areas. This paper reports on the present status of Solifugae in conserved areas. South Africa has a rich Solifugae fauna represented by 146 species. Of these species, 46 (31.5\%) represented by three families and 11 genera are presently protected in South African national parks, reserves or wilderness areas.
\end{abstract}

Key words: Arachnida, check list, national parks, Solifugae, South Africa, South African National Survey of Arachnida (SANSA).

A. S. Dippenaar-Schoeman, ARC-Plant Protection Research Institute, Private Bag X134, Queenswood, 0121 Republic of South Africa/Department of Zoology and Entomology, University of Pretoria, 0001 RSA; A.X. González Reyes, Crisóstomo Alvarex 2024, 3er Piso Departmento 10 CP. 4000, San Miguel de Tucumán, Tucumán Argentina.

\section{Introduction}

As part of the South African National Survey of Arachnida (SANSA) inventories are underway to determine the diversity of the South African Arachnida fauna (DippenaarSchoeman \& Craemer 2000). Several SANSA projects are in progress, including inventories of the arachnid faunas of protected areas. One such project is an inventory of the Solifugae (sun spiders) from protected areas. Meaningful conservation can not take place if the species involved are not known.

The Solifugae, an order of the Arachnida, are also known as sun-spiders, wind-scorpions, camel-spiders or romans. They are known from tropical, subtropical and arid environments in southern Europe, Africa, Asia and the Americas (Punzo 1998). A total of 1075 species from 12 families is presently known (Harvey 2003). South Africa has a rich fauna of sun-spiders, with 150 known species represented by six families (Dippenaar-
Schoeman 2002). This represents about $14 \%$ of the world's fauna. Of these species, $107(74 \%)$ are endemic to South Africa (DippenaarSchoeman 2002). Little is still known about the conservation status of the sun-spiders in South Africa and no red list is available. In an ealier paper on the fauna of South Africa, Lawrence (1955) indicates that some species have very restricted and localised distributions. This was supported by Griffin (1990), who found that most sun-spiders appear to have very restricted ranges and may be habitat dependent. Thus, it is important not only to consider the conservation of the sun-spiders alone, but also of their habitats, particularly in the case of locally distributed species.

The first inventory of Solifugae in a protected area was made by Lawrence (1964a, 1964b), who recorded 18 species from the Kruger National Park. Lamoral (1973) reported on six species known from the Kalahari Gemsbok National Park (now Kgalagadi Trans- 
Table 1

List of Solifugae species recorded from protected areas in South Africa.

\section{National Parks}

Augrabies National Park Biton (Biton) cataractus Lawrence, 1968 Blossia orangica (Lawrence, 1935a)* Blossia rosea (Lawrence, 1935a)* Hexisopus reticulatus Purcell, 1902*

Kgalagadi Transfrontier Park Biton (Biton) longisetosus Lawrence, 1972* Biton (Biton) schultzei (Kraepelin, 1908) Biton (Biton) tenuifalcis Lawrence, 1962 Blossia pallideflava (Lawrence, 1972)* Blossia rosea (Lawrence, 1935a)* Chelypus barberi Purcell, 1902 Chelypus hirsti Hewitt, 1915 Chelypus lennoxae Hewitt, 1912

Kruger National Park Biton (Biton) transvaalensis Lawrence, 1949* Blossia laticosta Hewitt, 1919 Blossia singularis (Lawrence, 1965)* Hemiblossia bouvieri Kraepelin, 1899 Solpuga conservatum (Lawrence, 1964)* Solpuga truncata (Lawrence, 1968)* Solpugema krugeri Lawrence, 1964c* Solpugiba arenicola Lawrence, 1964a Zeria celeripes (Hirst, 1911)

Zeria monteiri (Pocock, 1895)

Zeria sericea (Pocock, 1897)

Zeria serraticornis (Purcell, 1899)

Zeria spiralicornis (Purcell, 1903)

Zeria strepsiceros nocturna (Lawrence, 1965)*

Zeriassa furcicornis Lawrence, 1929*

Zeriassa purcelli Hewitt, 1914

Zeriassa transvaalensis Lawrence, 1964b* Zeriassa tuxeni Lawrence, 1965
Richtersveld National Park Biton (Biton) adamanteus polytricha Lawrence, $1972 *$

Biton (Biton) tenuifalcis Lawrence, 1962

Blossia clunigera Kraepelin, 1908*

Blossia namaquensis Purcell, 1902*

Solpugema genucornis (Lawrence, 1935b)*

Table Mountain National Park

Blossia litoralis Purcell, 1903a*

Solpuga villosa Purcell, 1899*

Solpugema vincta (C.L.Koch, 1842)*

Zeria fusca (C.L. Koch, 1842)*

Zeria schoenlandi (Pocock, 1900)

\section{Other Protected Areas}

Hluhluwe Game Reserve Solpuga suffusca Hewitt, 1916

Swartberg Nature Reserve Solpugema brachyceras (Lawrence, 1931)* Solpugema cycloceras (Lawrence, 1931)*

Grootvadersbosch Wilderness Area Solpugema phylloceras (Lawrence, 1929)*

Cedarberg Wilderness Area Solpuguna cervina (Purcell, 1899)*

Great Winterhoek Wilderness Area Zeria antelopicornis (Lawrence, 1929)*

Blyde River Nature Reserve Zeria carli (Roewer, 1933)*

* South African endemic

frontier Park) and four from the Augrabies National Park.

Solifugae are more common in warm and arid regions, and twice as many species are found in the western half of South Africa than in the east, with the highest numbers recorded from the Northern Cape Province (Wharton 1981; Dippenaar-Schoeman 2002). Most sunspiders appear to have very restricted ranges and may be habitat dependent (Dean \& Griffin 1993; Griffin 1990).
The sun-spiders are solitary animals with a strong antisocial nature. They live in retreats scraped out under rocks and logs. They use their chelicerae to loosen the soil and use their front body and legs to move large amounts of soil around. These retreats are used for resting, ecdysis and the deposition of eggs. They will defend themselves voraciously against their enemies. Because of a lack of venom they use speed and their strong chelicerae to attack. They prey on insects, other arachnids and even reptiles like geckos and lizards. 
Table 2

A check list of Solifugae from protected areas in South Africa. An * following a species name indicates a South African endemic

I. Family Daesiidae Kraepelin, 1899

i. Genus Biton Karsch, 1880

1. Biton (Biton) adamanteus polytricha Lawrence, 1972*

Lawrence, 1972: 107; Harvey, 2003: 219.

Type locality: $1.25 \mathrm{~km}$ E of Swartpoort (Swartport), Richtersveld, Northern Cape Province.

Park/reserve: Richtersveld National Park.

Distribution: South Africa.

2. Biton (Biton) cataractus Lawrence, 1968

Lawrence, 1968: 59; Wharton, 1981: 16; Harvey, 2003: 222.

Type locality: Augrabies Falls, Northern Cape Province.

Park/reserve: Augrabies National Park.

Distribution: South Africa, Namibia.

3. Biton (Biton) longisetosus Lawrence, 1972*

Lawrence, 1972: 106; Wharton, 1981: 18; Harvey, 2003: 220.

Type locality: Twee Rivieren, Kalahari Gemsbok National Park (now Kgalagadi Transfrontier Park), Northern Cape Province.

Park/reserve: Kgalagadi Transfrontier Park.

Distribution: South Africa.

4. Biton (Biton) schultzei (Kraepelin, 1908)

Kraepelin, 1908: 274 (from Daesia); Hewitt, 1919: 54; Lawrence, 1955: 186; 1959: 378; Wharton, 1981:

18; Harvey, 2003: 224.

Type locality: Khakhea, Botswana.

Park/reserve: Kgalagadi Transfrontier Park.

Distribution: Botswana, South Africa.

5. Biton (Biton) tenuifalcis Lawrence, 1962

Lawrence, 1962: 197; Wharton, 1981: 19; Harvey, 2003: 225.

Type locality: Twee Rivieren, Kalahari Gemsbok National Park (now Kgalagadi Transfrontier Park), Northern Cape.

Park/reserve: Kgalagadi Transfrontier Park, Richtersveld National Park.

Distribution: Namibia, South Africa.

6. Biton (Biton) transvaalensis Lawrence, 1949*

Lawrence, 1949: 204; 1955: 186; 1964b: 34; 1967: 83; Harvey, 2003: 225.

Type locality: Great Salt Pan, Waterpoort, Soutpansberg district, Limpopo Province.

Park/reserve: Kruger National Park (Letaba, Shingwedzi, Mahlakuza Pan).

Distribution: South Africa.

ii. Genus Blossia Simon, 1880

1. Blossia clunigera Kraepelin, 1908*

Kraepelin, 1908: 275; Hewitt, 1919a: 59; Lawrence, 1955: 179; Wharton, 1981: 25; Harvey, $2003: 213$.

Type locality: Steinkopf, Northern Cape Province.

Park/reserve: Richtersveld National Park (Wharton, 1981).

Distribution: South Africa.

2. Blossia laticosta Hewitt, 1919

Hewitt, 1919: 64, 59; Lawrence, 1964b: 34; 1955: 181; 1967: 83; Harvey, 2003: 216.

Type locality: Blauwkop, Soutpansberg District, Limpopo Province.

Park/reserve: Kruger National Park (Mahlakuza Pan, Orpen Camp, Pumbe, Punda Milia Rest Camp, Malelane Rest Camp, Pafuri, Krokodil Bridge).

Distribution: South Africa, Israel, Somalia? 
3. Blossia litoralis Purcell, 1903*

Purcell, 1903a: 4; Hewitt, 1919: 57; Lawrence, 1955: 156; 1962: 201; Harvey, 2003: 216.

Type locality: Duikerklip near Houtbay, Western Cape Province.

Park/reserve: Table Mountain National Park.

Distribution: South Africa.

4. Blossia namaquensis Purcell, 1902*

Purcell, 1902: 221; Hewitt, 1919: 56; Lawrence, 1955: 181; Wharton, 1981: 27; Harvey, 2003: 216.

Type locality: Steinkopf, Northern Cape Province.

Parks/reserves: Wharton (1981) recorded it from border of Richtersveld National Park at $28^{\circ} 19^{\prime}$ 'S; $16^{\circ} 58^{\prime} \mathrm{E}$.

Distribution: Namibia, South Africa.

5. Blossia orangica (Lawrence, 1935)*

Lawrence, 1935a: 78 (from Blossiola); 1955: 182; Wharton, 1981: 27; Harvey, 2003: 217.

Type locality: Augrabies Falls, Northern Cape Province.

Parks/reserves: Augrabies National Park.

Distribution: South Africa.

6. Blossia pallideflava (Lawrence, 1972)*

Lawrence, 1972: 99 (from Blossiola); Wharton, 1981: 27; Harvey, 2003: 217.

Type locality: Twee Rivieren, Kalahari Gemsbok National Park, Northern Cape Province.

Parks/reserves: Kgalagadi Transfrontier Park.

Distribution: South Africa.

7. Blossia rosea (Lawrence, 1935)*

Lawrence, 1935a: 76 (from Blossiola); 1955: 182; Wharton, 1981: 31; Harvey, 2003: 218.

Type locality: Augrabies Falls, Northern Cape Province.

Parks/reserves: Augrabies National Park; Kgalagadi Transfrontier Park (Twee Rivieren).

Distribution: South Africa.

8. Blossia singularis (Lawrence, 1965)*

Lawrence, 1965: 55 (from Blossiola); Harvey, 2003: 218.

Type locality: Kruger National Park (Shipale, Punda Maria area) Limpopo Province.

Parks/reserves: Kruger National Park.

Distribution: South Africa.

iii. Genus Hemiblossia Kraepelin, 1899

1. Hemiblossia bouvieri Kraepelin, 1899

Kraepelin, 1899: 233; Hewitt, 1919: 61; Lawrence, 1953: 959; 1955: 155; 1964b: 34; 1965: 68; Wharton, 1981: 36; Harvey, 2003: 232.

Type locality: Upper Zambezi River, Zambia?

Parks/reserves: Kruger National Park (Hapi Dam, Pafuri).

Distribution: Angola, Kenya, Namibia, South Africa, Zambia, Zimbabwe.

II. Family Hexisopodidae Pocock, 1879

i. Genus Chelypus Purcell, 1902

1. Chelypus barberi Purcell, 1902

Purcell, 1902: 224; Hewitt, 1919: 70; Lawrence, 1955: 173; 1959: 378; Lamoral, 1973: 91; Wharton, 1981: 46; Harvey, 2003: 280.

Type locality: Zandkuil, Northern Cape Province.

Parks/reserves: Kgalagadi Transfrontier Park (Mata Mata, Twee Rivieren).

Distribution: Angola, Botswana, Namibia, Zimbabwe, South Africa. 
2. Chelypus hirsti Hewitt, 1915

Hewitt, 1915: 323; 1919: 70; Lawrence, 1955: 173; 1963: 15; Lamoral, 1973: 90; Wharton, 1981: 46; Harvey, 2003: 281.

Type locality: Rietfontein, Northern Cape Province.

Parks/reserves: Kgalagadi Transfrontier Park (Twee Rivieren).

Distribution: Botswana, Namibia, South Africa.

3. Chelypus lennoxae Hewitt, 1912

Hewitt, 1912: 312; 1919: 70; Lawrence, 1955: 173; Lamoral, 1973: 90; Wharton, 1981: 46; Harvey, 2003: 281.

Type locality: Upington, Northern Cape Province.

Parks/reserves: Kgalagadi Transfrontier Park.

Distribution: Namibia, South Africa.

ii. Genus Hexisopus Karsch, 1879

1. Hexisopus reticulatus Purcell, 1902*

Purcell, 1902: 222; Hewitt, 1919: 69; Lawrence, 1955: 172; Wharton, 1981: 52; Harvey, 2003: 283.

Type locality: Between Bysteek and Great Falls at Augrabies, Northern Cape Province.

Parks/reserves: Augrabies National Park.

Distribution: South Africa.

III. Family Solpugidae Leach, 1816

i. Genus Solpuga Lichtenstein, 1796

1. Solpuga conservatum (Lawrence, 1964)*

Lawrence, 1964a: 529 (from Solpugopa); 1964b: 33; 1967; 38; Harvey, 2003: 307.

Type locality: between Saselandonga Poort and Mahlakuza Pan on eastern boundary, Kruger National Park.

Parks/reserves: Kruger National Park (between Saselandonga Poort and Mahlakuza Pan, between Mahlakuza and Malanga, Shangoni Koppies, Mwanedzi Dam, Skukuza).

Distribution: South Africa.

2. Solpuga suffusca Hewitt, 1916

Hewitt, 1916: 217; 1919: 32; Lawrence, 1955: 195; Harvey, 2003: 308.

Type locality: Hluhluwe Game Reserve, KwaZulu-Natal.

Parks/reserves: Hluhluwe Game Reserve.

Distribution: South Africa.

3. Solpuga truncata (Lawrence, 1968)*

Lawrence, 1968: 53 (from Solpugopa); Harvey, 2003: 309.

Type locality: between Shalungwa and Maseya, Kruger National Park.

Parks/reserves: Kruger National Park.

Distribution: South Africa.

4. Solpuga villosa Purcell, 1899*

Purcell, 1899: 422; Hewitt, 1919a: 41; Lawrence, 1955: 201; Harvey, 2003: 309.

Type locality: No exact locality only labelled "S.Africa".

Parks/reserves: Tafelberg National Park.

Distribution: South Africa.

ii. Genus Solpugema Roewer, 1933

1. Solpugema brachyceras (Lawrence, 1931)*

Lawrence, 1931: 132 (from Solpuga); 1955: 197; Harvey, 2003: 311.

Type locality: Swartberg Pass, near Oudtshoorn, Western Cape Province.

Parks/reserves: Swartberg Nature Reserve.

Distribution: South Africa. 
2. Solpugema cycloceras (Lawrence, 1931)*

Lawrence, 1931: 131 (from Solpuga); 1955: 198; Harvey, 2003: 311.

Type locality: Swartberg Pass, near Oudtshoorn, Western Cape Province.

Parks/reserves: Swartberg Nature Reserve.

Distribution: South Africa.

3. Solpugema genucornis (Lawrence, 1935)*

Lawrence, 1935b: 505 (from Solpuga); 1955: 198; Wharton, 1981: 81; Harvey, 2003: 311.

Type locality: Lekkersing, Richtersveld, Northern Cape Province.

Parks/reserves: Richtersveld National Park.

Distribution: Namibia, South Africa.

4. Solpugema krugeri Lawrence, 1964*

Lawrence, 1964c: 355; 1964b: 33; Harvey, 2003: 313.

Type locality: Banks of Sabie River, Kruger National Park.

Parks/reserves: Kruger National Park.

Distribution: South Africa.

5. Solpugema phylloceras (Lawrence, 1929)*

Lawrence, 1929: 164; 1955; 200; Harvey, 2003: 313.

Type locality: Pocaltsdorp near George, Western Cape Province.

Parks/reserves: Grootvadersbosch Wilderness Area.

Distribution: South Africa.

6. Solpugema vincta (C.L.Koch, 1842)*

C.L. Koch, 1842: 352 (as Solpuga); Hewitt, 1919: 33; Lawrence, 1929: 178; 1955: 200; Harvey, 2003:

314.

Type locality: no exact locality only "Cap".

Parks/reserves: Table Mountain National Park.

Distribution: South Africa.

iii. Genus Solpugiba Roewer, 1934

1. Solpugiba arenicola Lawrence, 1964

Lawrence, 1964a: 1964b: 33; 1967: 84; Harvey, 2003: 314.

Type locality: Between Shalungwa and Maseya, Kruger National Park.

Parks/reserves: Kruger National Park (Sandveld between Shalungwa and Maseya, 1 km S of Tambye Drift near Pafuri, Skukuza Rest Camp, Isweni near Satara).

Distribution: South Africa.

iv. Genus Solpuguna Roewer, 1933

1 Solpuguna cervina (Purcell, 1899)*

Purcell, 1899: 415; Hewitt, 1919: 32; Lawrence, 1964a: 531; 1964b; Wharton, 1981: 75; Harvey, 2003:

316.

Type locality: Clanwilliam, Western Cape Province.

Parks/reserves: Cedarberg Wilderness Area.

Distribution: South Africa.

v. Genus Zeria Simon, 1879

1. Zeria antelopicornis (Lawrence, 1929)*

Lawrence, 1929: 153 (from Solpuga); 1955: 191; Harvey, 2003: 317.

Type locality: Great Winterhoek Mountains, Western Cape Province.

Parks/reserves: Great Winterhoek Wilderness Area.

Distribution: South Africa.

2. Zeria carli (Roewer, 1933)*

Roewer, 1933: 461 (from Solpuga); Lawrence, 1955: 192; Harvey, 2003: 318.

Type locality: near Johannesburg, Gauteng Province. 
Parks/reserves: Blyde River Nature Reserve.

Distribution: South Africa.

3. Zeria celeripes (Hirst, 1911)

Hirst, 1911: 10 (from Solpuga); Hewitt, 1919: 31; Lawrence, 1929: 178; 1955: 192; 1964b: 34; 1965: 49; 1967: 82; Harvey, 2003: 318.

Type locality: Harare, Zimbabwe.

Parks/reserves: Kruger National Park (Tswiriridam, Nwanetsani Dam, Saselandonga Poort, Mahlakuza

Pan, Pretoriuskop, Tshokwane, Pumbe Picket)

Distribution: Zimbabwe, South Africa.

4. Zeria fusca (C.L. Koch, 1842)*

C.L. Koch, 1842: 352 (from Solpuga); Hewitt, 1919: 26; Lawrence, 1929: 179; 1955: 166; Harvey, 2003: 319.

Type locality: "Cap" no exact locality.

Parks/reserves: Table Mountain National Park.

Distribution: South Africa.

5. Zeria monteiri (Pocock, 1895)

Pocock, 1895: 87 (from Solpuga); Hewitt, 1919: 27; 1935: 476; Lawrence, 1928: 262; 1929: 178; 1949: 208; 1953: 969; 1955; 193; 1964b: 33; 1967: 83.

Type locality: Maputo, Mozambique.

Parks/reserves: Kruger National Park (Letaba Camp, Shingwedzi, Skukuza).

Distribution: Angola, Botswana, Mozambique, Namibia, Zimbabwe, South Africa.

6. Zeria schoenlandi (Pocock, 1900)

Pocock, 1900: 316 (as Solpuga); Hewitt, 1919: 29; Lawrence, 1955: 194; Wharton, 1981: 78; Harvey, 2003: 323 .

Type locality: Grahamstown, Eastern Cape Province.

Parks/reserves: Table Mountain National Park.

Distribution: South Africa, Zimbabwe.

7. Zeria sericea (Pocock, 1897)

Pocock, 1897: 260 (from Solpuga); Lawrence, 1955: 194; 1964b: 33; 1967: 83; Wharton, 1981: 78;

Harvey, 2003: 323.

Type locality: Gadzema, Zimbabwe.

Parks/reserves: Kruger Nacional Park (Punda Milia area, Shilowane, Madziringwe Poort, Saselandonga

Poort, Sandveld, Sabie Hoek).

Distribution: Zimbabwe, Democratic Republic of the Congo, South Africa.

8. Zeria serraticornis (Purcell, 1899)

Purcell, 1899: 409 (from Solpuga); Lawrence, 1955: 194; 1967: 83; Harvey, 2003: 323.

Type locality: Bulawayo, Zimbabwe.

Parks/reserves: Kruger National Park (Msimbit Forest, eastern boundary of Pafuri).

Distribution: Zimbawbe, Ethiopia, Mozambique, Democratic Republic of the Congo, South Africa.

9. Zeria spiralicornis (Purcell, 1903)

Purcell, 1903b: 304 (from Solpuga); Lawrence, 1955: 194; 1964b: 33; 1967: 83; Harvey, 2003; 323.

Type locality: Shilouvane, near Kranskop, KwaZulu-Natal Province.

Parks/reserves: Kruger National Park (Shingwedzi, Olifants Camp, Skukuza, Malelane Rest Camp,

Shalungwa Springs, Satara Rest Camp).

Distribution: Zimbabwe, South Africa.

10. Zeria strepsiceros nocturna (Lawrence, 1965)*

Lawrence, 1965: 51 (from Solpuga): 1967: 83; Harvey, 2003: 324.

Type locality: Orpen Camp, Kruger National Park.

Parks/reserves: Kruger National Park (Orpen Camp, Mtsawu Drift, Doispanne road, Sitlabe Dam, Sabi

Poort, Krokodil Bridge).

Distribution: South Africa. 
vi. Genus Zeriassa Pocock, 1897

1. Zeriassa furcicornis Lawrence, $1929^{*}$

Lawrence, 1929: 169; 1955: 188; 1964b: 31; 1967: 82; Harvey, 2003: 325.

Type locality: Kaapmuiden, Mpumalanga Province.

Parks/reserves: Kruger National Park (Shingwezdi, Pafuri, Nyandu Bush, Shalungwa Springs, Punda

Milia Rest Camp).

Distribution: South Africa.

2. Zeriassa purcelli Hewitt, 1914

Hewitt, 1914: 163; Hewitt, 1919: 52; Lawrence, 1955: 164; Harvey, 2003: 326.

Type locality: Newington, Limpopo Province.

Parks/reserves: Kruger National Park (Leeupan, Skukuza).

Distribution: South Africa.

3. Zeriassa transvaalensis Lawrence, 1964*

Lawrence, 1964b: 31; 1967: 83; Harvey, 2003: 327.

Type locality: Tshokwane tea room, Skukuza, Kruger National Park.

Parks/reserves: Kruger National Park (Orpen Camp, Mahlakuza Pan, Malanga, Leeupan, Krokodil

Bridge, Malelane Camp, Skukuza Rest Camp).

Distribution: South Africa.

4. Zeriassa tuxeni Lawrence, 1965

Lawrence, 1965: 48; Harvey, 2003: 327.

Type locality: Letaba Camp, Kruger National Park.

Parks/reserves: Kruger National Park (Letaba Camp).

Distribution: South Africa.

Some species are exclusively predators of termites (Cloudsley-Thompson 1977; Dippenaar-Schoeman 1993; Punzo 1998).

The aim of this paper is to provide an updated check list of the known Solifugae species that have so far been recorded from protected areas (national parks, nature reserves and wilderness areas) in South Africa to show the percentage of species presently protected.

\section{Method}

Data on the occurrence and distribution of the species were obtained from published literature and material housed in several collections. Species known to be endemic to South Africa are indicated with an asterisk in Tables 1 and 2. Only literature references relative to the areas under discussion were listed; for detailed references on each species see Harvey (2003). The latest nomenclature based on Harvey (2003) was used to compile the updated check list.

\section{Discussion}

The Solifugae presently known from national parks and reserves of South Africa are represented by three families, 11 genera and 46 species (Table 1 ).

The 46 species represent $30.7 \%$ of the South African fauna that might be presently protected on conserved areas. However, more surveys are needed to increase the sampling effort and improve the knowledge of the distribution of particular species. Also, most of the specimens examined were collected between 30-100 years ago, and therefore, new surveys are needed as part of SANSA to provide more current information on species within protected areas. There is also an urgent need for taxonomic support 
to improve the reliability of the biological databases on Solifugae as the last taxonomic research on the South African fauna was undertaken by Lawrence in the late 1960s, and by Lamoral (1973).

\section{References}

Cloudsley-Thompson, J.L. 1977. Adaptational biology of Solifugae (Solpugida). Bulletin of the British Arachnological Society 4: 61-71.

DEAN, W.R.J. \& E. GRIFFIN. 1993. Seasonal activity patterns and habitats in Solifugae (Arachnida) in the southern Karoo, South Africa. South African Journal of Zoology 28: 91-94.

DipPENAAR-SchOEMAN, A.S. 1993. Sun-spiders, some interesting facts. African Wildlife 47: 120-122.

DipPenaAR-Schoeman, A.S. 2002. Status of South African Arachnida Fauna. Pp. 70-81. In: VeRDOORN, G.H. \& J. LE Roux (eds). The State of South Africa's species. Proceedings of a conference held at the Rosebank Hotel, 4-7 September 2001.

DippenaAR-Schoeman, A.S. \& C. Craemer. 2000. The South African National Survey of Arachnida (SANSA). Plant Protection News 56: 11-12.

GrIFFIN, E. 1990. Seasonal activity, habitat selection and species richness of Solifugae (Arácnida) on the gravel plain of the central Namib Desert. Transvaal Museum Monograph 7: 77-82.

HaRveY, M.S. 2003. Catalogue of the smaller arachnid orders of the World. Australia: CSIRO publishing.

HewitT, J. 1912. Description of a new species of Chelypus (Solpugidae). Records of the Albany Museum 2: 312-313.

HewitT, J. 1914. Records of species of Solifugae in the collection of the Transvaal Museum and descriptions of several new species of the family Solpugidae. Annals of the Transvaal Museum 4: 160-167.

Hewitt, J. 1915. New South African Arachnida. Annals of the Natal Museum 3: 289-327.

HewitT, J. 1916. Description of several species of Arachnida in the collection of the Durban Museum. Annals of the Durban Museum 3: 70-106.

HewitT, J. 1919. A short survey of the Solifugae of South Africa. Annals of the Transvaal Museum 7: 1-76.

HewitT, J. 1935. Scientific results of the Vernay-Lang Kalahari Expedition, March to september, 1930. The trap-door spiders, scorpions and solifuges. Annals of the Transvaal Museum 16: 459-479.

HIRST, S. 1911. On a collection of Arachnida and Chilopoda, made by Mr. S.A. Neave in Rhodesia, north of the Zambezi. Memoirs and Proceedings of Manchester Literary and Philosophical Society 56: 1-11.

Kосн, C.L. 1842. Systematische Uebersicht über die familie der Galeoden. Archiv für Naturgeschichte 8: 350-356.

Kraepelin, K. 1899. Zur Systematik der Solifugen. Mitteilungen aus dem Naturhistorischen Museum in Hamburg 16: 195-258.

KraePelin, K. 1908. Skorpione und Solifuges. In: Schultze, L. (ed.) Forschungsreise im westlichen und zentralen Südafrika 13: 247-282. G. Fischer Jena.

LAMORAL, 1973. The Arachnid fauna of the Kalahari Gemsbok National Park Part. 1. A revision of the species of "mole solifuges" of the genus Chelypus Purcell, 1901 (family Hexisopodidae). Koedoe 16: 83-102.

LaWrence, R.F. 1929. New South African Solifugae. Annals of the South African Museum 29: 153-179.

LaWrence, R.F. 1931. New South African Solifugae. Annals of the South African Museum 30: 131-136.

Lawrence, R.F. 1935a. New South African Solifugae. Transactions of the Royal Society of South Africa 23: 71-90.

LAWRENCE, R.F. 1935b. New species of Solifugae in the collection of the Transvaal Museum. Annals of the Transvaal Museum 15: 505-512

LAWRENCE, R.F. 1949. New species of Solifugae in the collection of the Transvaal Museum. Annals of the Transvaal Museum 21: 201-208.

LAWRENCE, R.F. 1953. A collection of African Solifugae in the British Museum (Natural History). Proceedings of the Zoological Society of London 122: 955-972

LaWrence, R.F. 1955. Solifugae, Scorpions and Pedipalpi, with checklists and keys to South African families, genera and species. South African Animal Life 1: 152-262.

LAWRENCE, R.F. 1959. A collection of Arachnida and Myriapoda from the Transvaal Museum. Annals of the Transvaal Museum 23: 363-386.

LaWrence, R.F. 1962. A new South African Solifugae (Arachnida) with notes on some known species. Transaction of the Royal Society of South Africa 36: 197-203.

LAWRENCE, R.F. 1964a. New Solifugae (Arachnida) from the Kruger National Park, South Africa. Annals and Magazine of Natural History 13: 529-535.

LaWrenCE, R.F. 1964b. The Solifugae, Scorpions and Pedipalpi of the Kruger National Park. Koedoe 7: 30-39.

LAWRENCE, R.F. 1964c. Four new South African Solifugae (Arachnida). Journal of the Entomological Society of Southern Africa 26: 355-365.

LAWRENCE, R.F. 1965. Some new or little known Solifugae from Southern Africa. Proceedings of the Zoological Society in London 144: 47-59. 
LAWRENCE, R.F. 1967. Supplementary list of the Solifugae, Scorpions and Pedipalpi of the Kruger National Park. Koedoe 10: 82-86.

LAWRENCE, R.F. 1968. A contribution to the solifugid fauna of southern Africa (Arachnida). Annals of the Transvaal Museum 26: 53-77.

LAWRENCE, R.F. 1972. New psammophilous Solifugae, chiefly from desert regions of the Kalahari and South West Africa. Madoqua series II: 97-116.

Pocock, R.I. 1895. Notes on some of the Solifugae contained in the collection of the British Museum, with descriptions of new species. Annals and Magazine of Natural History (6) 16: 74-98.

Pocock, R.I. 1897. On the genera and species of tropical African Arachnida of the order Solifugae, with notes upon the taxonomy and habits of the group. Annals and Magazine of Natural History 6(20): 249-272.

Pocock, R.I. 1900. Some new Arachnida from the Cape Colony. Annals and Magazine of Natural History 7(6): 316-332

Punzo, F. 1998. The biology of camel-spiders (Arachnida, Solifugae). Boston: Kluwer Academic Publishers.
Purcell, W.F. 1899. New and little known South African Solifugae in the collection of the South African Museum. Annals of the South African Museum 1: 381-432.

PurCELl, W.F. 1902. On some South African Arachnida belonging to the orders Scorpiones, Pedipalpi, and Solifugae. Annals of the South African Museum 2: 137-225.

PurCELL, W.F. 1903a. Descriptions of new genera and species of South African Solpugidae. Annals of the South African Museum 3: 1-12.

PURCELL, W.F. 1903b. On the scorpions, solifugae, and a trapdoor spider, collected by Rev. Henri A. Junod, at Shilouvane, near Leydsdorp, in the Transvaal. Novitates Zoologicae, London 10: 303-306.

Roewer, C.F. 1933. Solifugae, palpigradi. Pp. 161480. In: BonN H.G. (ed.). Klassen und Ordnungen des Tierreichs. 5. Arthropoda. IV. Arachnoidea. Leipzig: Akademische Verlagsgesellschaft M.B.H.

Wharton, R.A. 1981. Namibian Solifugae (Arachnida). Cimbebasia Memoir 5: 1-87. 